\title{
Association between Vascular Endothelial Growth Factor and Clinical Outcomes of IVF-ET/ICSI
}

\author{
Hui Chen, Jian-Bo Zheng, Dong-Mei Wang, Hua Xing and Hui Wang
}

\begin{abstract}
Objective: To investigate the association between the level of vascular endothelial growth factor (VEGF) and the quality of early embryos as well as clinical outcome after embryo transplantation in embryo culture medium of patients treated with IVF-ET/ICSI, and explore the association between VEGF $936 \mathrm{C} / \mathrm{T}$ gene polymorphisms and IVF-ET/ICSI clinical outcomes.

Study Design: Case-control study.

Place and Duration of Study: The Reproductive Medical Center, Yantaishan Hospital, Yantai, Shandong Province, China, from January to December 2016.

Methodology: Ninety-eight patients undergoing IVF-ET/ICSI treatment were divided into pregnant group ( $\mathrm{n=58}$ ) and nonpregnant group $(n=40)$, based on clinical outcomes. Average age, infertility duration, body mass index, number of previous grafts, basic follicle-stimulating hormone (FSH) levels, luteinizing hormone (LH) levels, chorionic gonadotropin (HCG) intimal thickness, estrogen (E2) levels, progesterone $(P)$ levels, and other relevant indicators were compared between the two groups. The vascular endothelial growth factor (VEGF) levels in the embryo culture medium were detected by enzyme-linked immunosorbent assay (ELISA). Meanwhile, VEGF $936 \mathrm{C} / \mathrm{T}$ genotype and allele frequency (\%) were compared between the two groups.

Results: There was no significant difference in average age, infertility duration, number of previous grafts, basic folliclestimulating hormone (FSH) levels, luteinizing hormone (LH) levels, chorionic gonadotropin (HCG) intimal thickness, estrogen (E2) level, progesterone $(P)$ level, number of retrieved oocytes, embryo number, quality embryo number, quality embryo rate, and cumulative embryo score (CES) of transplanted embryo between the two groups $(P=0.601,0.180,0.741$, $0.119,0.693,0.282,0.064,0.505,0.712,0.822,0.663,0.150,0.178,0.204$ and 0.263 , respectively). VEGF level in embryo culture medium was lower in pregnant group than non-pregnant group, with statistically significant difference ( $<<0.001)$. VEGF level in the culture medium of grade I embryos was significantly lower than that of grade II embryos $(p<0.001)$. The frequencies of CC genotype and C allele in VEGF 936 in the non-pregnant group were higher than those in the pregnant group ( $p=0.003$, and 0.005 , respectively).

Conclusion: VEGF level in the embryo culture medium can be used as one reference indicator for predicting embryo quality and pregnancy outcome. The VEGF $936 \mathrm{C} / \mathrm{T}$ gene polymorphism may be related to IVF-ET/ICSI treatment outcome, and $C$ allele may be a susceptibility gene for IVF-ET/ICSI failure.
\end{abstract}

Key Words: Vascular endothelial growth factor, Vascular endothelial growth factor gene, Genotype, Embryo quality, In vitro fertilisation-embryo transplantation, Intracytoplasmic sperm injection.

\section{INTRODUCTION}

Vascular endothelial growth factor (VEGF) is the most important angiogenine discovered so far and is a growth factor yet known to have both pro-angiogenesis and vascular permeability enhancing functions in all proangiogenic factors. 1,2 Previous studies have shown that, in the ovulation cycle, with the follicle growth and maturation, VEGF levels in serum and follicular fluid were also significantly higher, suggesting that VEGF may participate in the development of follicles.3,4 However, there are few reports on the related research

Reproductive Medical Center, Yantaishan Hospital, Yantai, Shandong Province, 264000, China

Correspondence: Hui Chen, Reproductive Medical Center, Yantaishan Hospital, Yantai, Shandong Province, 264000, China

E-mail: atr6fu@163.com

Received: March 19, 2018; Accepted: August 13, 2018 of VEGF in human embryonic development. VEGF gene polymorphisms are associated with recurrent miscarriage, preeclampsia and premature birth.5,6 However, few studies have been reported on the association between VEGF gene polymorphisms and IVF-ET/ICSI outcomes. Relationship between vascular endothelial growth factor (VEGF) $936 \mathrm{C} / \mathrm{T}$ gene polymorphisms and IVF-ET/ICSI treatment outcomes can be studied by detection of serum VEGF genotypes in the pregnant and non-pregnant women, thus providing reference for IVF - ET/ICSI therapy.

The aim of this study was to analyse the association between VEGF levels in embryo culture mediums and clinical outcomes after embryo transplantation.

\section{METHODOLOGY}

This study was done in The Reproductive Medical Center, Yantaishan Hospital, Yantai, Shandong Province, China, from January to December 2016. All patients met 
the IVF-ET/ICSI indications and were Chinese Han women. This study was approved by the Hospital Ethics Committee and the patients signed informed consent. Using the unit's conventional ovulation-promoting programme to promote ovulation. Oocyte pickup was carried out under transvaginal B ultrasound guidance. Conventional in-vitro fertilisation was performed after seminal fluid treatment. Fertilisation was observed at 19hour after addition of sperm. After fertilisation, the fertilised ovums were transferred to embryonic culture droplets, with one culture droplet for one embryo. The embryos were incubated at $37^{\circ} \mathrm{C}$ in a $6 \% \mathrm{CO}_{2}$ incubator and observed after 48 hours for embryo scoring. The best two embryos were chosen for transplantation. Embryo culture medium was loaded as per 2030 $\mu \mathrm{L} / \mathrm{drop}$ after transfer of embryos and stored at $-80^{\circ} \mathrm{C}$ ultra-low temperature refrigerator for testing.

On the 3rd day of culture, the embryos were scored. According to the number of blastomeres, whether the cells were homogenous and whether there were debris, embryos were graded in line with Wetzels embryo scoring method. Grade I embryos (4 points) had uniform cells, regular shape, intact zona pellucid, even and clear cytoplasm, with cell debris at $5 \%$. Grade II embryos (3 points) had non-uniform cell size, slightly irregular shape, granule in cytoplasm, with cell debris between $10-20 \%$. Grade III embryos (2 points) had obviously uneven cell size, obviously irregular shape, granule in cytoplasm, with cell debris between $21-50 \%$. Grade IV embryos (1 point): extremely uneven cell, severe granule in cytoplasm, with cell debris above $50 \%$. Grade V embryos ( 0 point) were degenerated, dead embryos. The score for each embryo was obtained by multiplying embryonic morphological score with number of embryonic cells, and cumulative embryo score (CES) of each patient's transfer cycle was obtained by totaling the transplanted embryos' score in the transfer cycle.

According to clinical outcomes, all the 98 patients were divided into clinical pregnant group and non-pregnant group. The diagnosis of clinical pregnancy is ultrasound examination at 4 weeks after embryo transfer, seeing gestational sac and fetal buds, original heart tube or seeing villus at uterine curettage.

VEGF concentration in the culture medium was measured by ELISA. The reagent used for the assay was a VEGF kit and a pre-test was conducted to adjust the dilution ratio.

For all subjects, $5 \mathrm{~mL}$ of fresh peripheral venous whole blood was collected under aseptic conditions and anticoagulated with ethylenediaminetetraacetic acid. The specific operation of genomic DNA extraction was performed in strict accordance with the kit instructions. Polymerase chain reaction-restriction fragment length polymorphism (PCR-RFLP) technology was used to perform PCR amplification with white blood cell genomic
DNA in peripheral blood as a template. The upstream primer of VEGF $936 \mathrm{C} / \mathrm{T}$ was 5'-AAGGAAGA GGAGACTCTGCGCAGAGC-3', and the downstream primer was 5'-TAAATGTATGTGGGTGGGTGTGTC TACAGG-3'. The $50 \mu \mathrm{L}$ total reaction system contained one $\mu \mathrm{L}$ of upstream and downstream primers respectively, and approximately one $\mu \mathrm{L}$ of genomic DNA, and $25 \mu \mathrm{L} 2 \times \mathrm{HiFi}-\mathrm{PCR}$ Master $\left(3 \mathrm{mmol} / \mathrm{L} \mathrm{MgCl}_{2}, 0.2\right.$ $\mathrm{mmol} / \mathrm{L}$ dNTP, $0.1 \mathrm{U} / \mu \mathrm{L}$ Taq DNA polymerase, $2 x \mathrm{PCR}$ buffer), which was complemented by double distilled water. Amplification was performed on a PCR thermocycler: pre-denaturation at $94^{\circ} \mathrm{C}$ for 8 minutes, denaturation at $94^{\circ} \mathrm{C}$ for 30 seconds, annealing at $65^{\circ} \mathrm{C}$ for 30 seconds, extension at $72^{\circ} \mathrm{C}$ for 30 seconds, 30 cycles and final extension at $72^{\circ} \mathrm{C}$ for 10 minutes. The $15 \mu \mathrm{L}$ of the PCR reaction product was digested with restriction enzyme Nlalll $1 \mathrm{~L}$. The digested products were electrophoresed on $1.2 \%$ agarose, stained with ethidium bromide, observed under a UV lamp and photographed. The 208 bp 1 band was observed for TT genotype enzyme-free cutting site; the Nlalll restriction enzyme cutting site of CC genotype was cut into122 bp and 86 bp bands. The CT genotype part was cut, and 208, 122 and 86 bp bands were observed.

SPSS 21.0 statistical software package was used for data processing. Measurement data are expressed as mean \pm standard deviation, count data are expressed as frequencies with percentages. Measurement data are tested with independent-sample t-test, and count data are tested with Chi-square test. $\mathrm{P}<0.05$ indicates statistically significant differences.

\section{RESULTS}

Ninety-eight patients undergoing IVF-ET/ICSI treatment were selected, including $46(46.94 \%)$ cases of primary infertility and $52(53.06 \%)$ cases of secondary infertility.

Table I: Comparison of relevant indicators of patients with different clinical outcomes $(\chi \pm s)$.

\begin{tabular}{l|c|c|c}
\hline Index & $\begin{array}{c}\text { Pregnant group } \\
(\mathrm{n}=58)\end{array}$ & $\begin{array}{c}\text { Non-pregnant } \\
\text { group }(\mathrm{n}=40)\end{array}$ & $\mathrm{p}$ \\
\hline Female age (year) & $34.37 \pm 3.89$ & $34.84 \pm 4.96$ & 0.601 \\
\hline Infertility duration (year) & $4.57 \pm 0.50$ & $4.43 \pm 0.51$ & 0.180 \\
\hline Body mass index & $20.98 \pm 1.26$ & $20.87 \pm 2.03$ & 0.741 \\
\hline Number of previous grafts & $1.14 \pm 0.25$ & $1.21 \pm 0.15$ & 0.119 \\
\hline Basic FSH (mlU/mL) & $7.09 \pm 0.38$ & $7.06 \pm 0.35$ & 0.693 \\
\hline Basic LH (mIU/mL) & $4.69 \pm 0.39$ & $4.82 \pm 0.76$ & 0.282 \\
\hline Basic E2 (pg/mL) & $46.07 \pm 1.29$ & $46.91 \pm 3.04$ & 0.064 \\
\hline Chorionic gonadotropin $(\mathrm{HCG})$ & $7.43 \pm 0.84$ & $7.27 \pm 1.52$ & 0.505 \\
\hline intimal thickness (cm) & $3693.35 \pm 167.54$ & $3681.06 \pm 151.91$ & 0.712 \\
\hline Estrogen (E2) level, (pg/mL) & $1.33 \pm 0.41$ & $1.31 \pm 0.46$ & 0.822 \\
\hline Progesterone (P) level $(\mathrm{ng} / \mathrm{mL})$ & $19.46 \pm 4.19$ & $19.07 \pm 4.56$ & 0.663 \\
\hline Number of retrieved oocytes & $10.57 \pm 2.09$ & $9.89 \pm 2.53$ & 0.150 \\
\hline Embryo number & $7.15 \pm 0.84$ & $6.75 \pm 1.32$ & 0.070 \\
\hline Quality embryo number & $0.75 \pm 0.06$ & $0.73 \pm 0.10$ & 0.204 \\
\hline Quality embryo rate $(\%)$ & $43.36 \pm 4.61$ & $42.25 \pm 5.06$ & 0.263 \\
\hline Cumulative embryo score (Score) & $11.35 \pm 1.88$ & $14.08 \pm 2.63$ & $<0.0001$ \\
\hline VEGF (pg/mL) & & &
\end{tabular}


Table II: Distribution of VEGF genotypes and allele frequencies of the two groups [n (\%)]

\begin{tabular}{|c|c|c|c|c|c|c|}
\hline \multirow[t]{2}{*}{ Groups } & \multirow[t]{2}{*}{ Number of cases } & \multicolumn{3}{|c|}{ Genetype } & \multicolumn{2}{|c|}{ Allelomorph } \\
\hline & & $\mathrm{CC}$ & CT & TT & $\mathrm{C}$ & $\mathrm{T}$ \\
\hline Pregnant group & 58 & $37(63.79)$ & $16(27.59)$ & $5(8.62)$ & $94(81.03)$ & $22(18.97)$ \\
\hline Non-pregnant group & 40 & $36(90.00)$ & $3(7.50)$ & $1(2.50)$ & $76(95.00)$ & $4(5.00)$ \\
\hline$p$ & & \multicolumn{3}{|c|}{0.003} & \multicolumn{2}{|c|}{0.005} \\
\hline
\end{tabular}

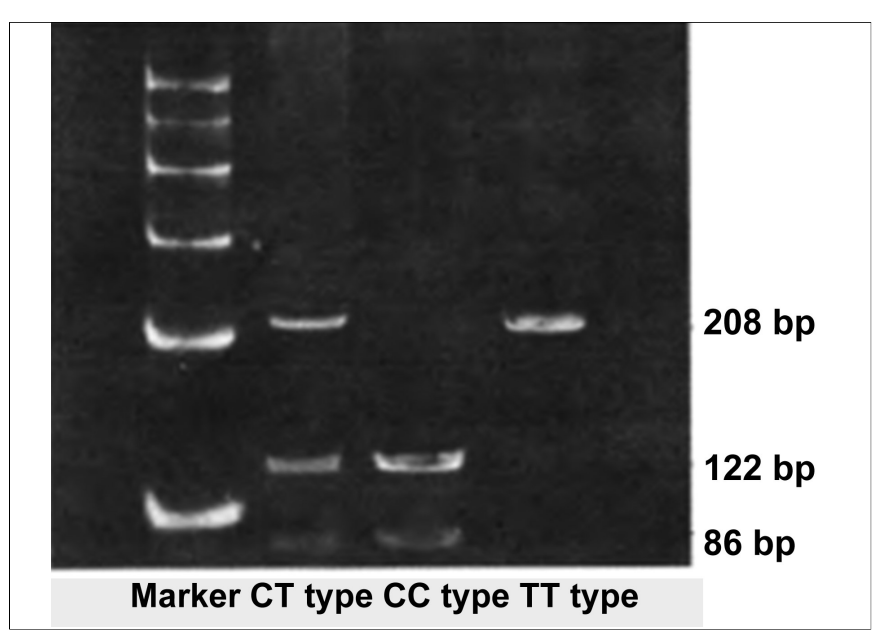

Figure 1: VEGF $936 \mathrm{C} / \mathrm{T}$ gene polymorphism.

Ranging from 24 to 48 years, the patients had an average age of $34.56 \pm 2.41$ years.

Of the 98 cases of embryo transplanted, $58(59.18 \%)$ cases got pregnant, making $58(59.18 \%)$ cases in the pregnant group and $40(40.82 \%)$ cases in the nonpregnant group. There were no significant differences in average age, infertility duration, body mass index, number of previous grafts, basic follicle-stimulating hormone (FSH) levels, luteinizing hormone (LH) levels, chorionic gonadotropin (HCG) intimal thickness, estrogen (E2) level, progesterone $(P)$ level, number of retrieved oocytes, embryo number, quality embryo number, quality embryo rate, and cumulative embryo score (CES) between the two groups. VEGF level in embryo culture medium of pregnant group was lower than that of non-pregnant group $(p<0.001$, Table I).

VEGF level in the culture medium of grade I embryo (4 points) was $8.03 \pm 0.76 \mathrm{pg} / \mathrm{mL}$, and that of grade II embryos (3 points) was $17.12 \pm 3.04 \mathrm{pg} / \mathrm{mL}$. VEGF level in the culture medium of grade I embryos with better implantation potential is lower than that of slightly deficient grade II embryos, the difference is statistically significant $(p<0.001)$.

Both pregnant and non-pregnant groups' VEGF genes have $\mathrm{C}$ and $\mathrm{T}$ alleles and CC, CT and TT genotypes. The distribution was consistent with Hardy-Weinberg equilibrium method (Figure 1). The frequencies of CC genotypes of VEGF 936 in non-pregnant and pregnant group were $90.0 \% \quad(n=36)$ and $63.79 \% \quad(n=37)$, respectively. $C$ allele frequencies were $95.00 \%(n=76)$ and $81.03 \%(n=94)$, respectively. The frequencies of CC genotype and $C$ allele in the non-pregnant group are higher than those in the pregnant group $(p=0.003$, and 0.005 , respectively, Table II).

\section{DISCUSSION}

Embryo quality is a key factor in the success of pregnancy. In the current human assisted reproductive technology, morphological parameters of embryos are still the main non-invasive indicators for embryo selection. 7,8 Although morphological assessment provides a simple and effective method for selection of embryos for transplantation, many quality embryos as assessed by morphological methods, failed to implant after transplantation.9,10 At present, there are many problems in embryo morphological assessment, such as the lack of quantifiable indicators and subjective factors of laboratory technicians. ${ }^{11-13}$ Therefore, how to increase prediction method and improve the ability to predict the potential of early embryo development has become a top priority. The assessment of embryonic developmental potential by measuring embryonic endogenous factors in embryonic metabolites may become an effective method for assessing embryo quality due to its non-invasive nature and low risk. ${ }^{14,15}$ Relevant data show that after removal of VEGF in the process of mouse embryo culture in-vitro, embryo growth retarded or even stopped. ${ }^{16}$ But returned to normal and showed cardiovascular formation after VEGF was added. However, there are few reports on the related research of VEGF in human embryonic development.

Previous studies have suggested that clinical pregnancy of in-vitro fertilisation (IVF) is related to embryo quality and endometrial receptivity. ${ }^{17}$ However, the results of this study show that there was no difference in clinical characteristics (average age, infertility duration, gonadotropin dosage, number of gonadotropins, and HCG intimal thickness) and embryonic development (number of received oocytes, number of embryos, number of quality embryos, and the cumulative embryo score of transplanted embryos) between the two groups. At the same time, expression levels of VEGF in embryo culture medium of pregnant group and non-pregnant group were initially compared. The result showed that VEGF level in embryo culture medium of pregnant group was lower than that of non-pregnant group, suggesting that quality embryos with low VEGF levels have a high ability to grow. At the same time, we also found that VEGF level in the culture medium of grade I embryos with better implantation potential was lower than that of 
slightly deficient grade II embryos. The difference is statistically significant $(p<0.001)$. All these results suggest that VEGF levels in embryo culture medium may predict clinical outcomes after embryo transfer earlier and more sensitively than embryo morphology and may serve as a reference indicator for predicting pregnancy outcomes.

Gene polymorphism refers to differences in nucleotide sequences between individuals. Polymorphisms occur at gene level mutations. There may be more than two genotypes in the same gene locus, which is prevalent in areas with noncoding protein or without important adjustment functions in gene sequences. ${ }^{18}$ The human VEGF gene is located on long arm of chromosome 6p21.3. The gene encoding VEGF is about $14 \mathrm{~Kb}$ in length and is composed of 8 exons and 7 introns alternately. ${ }^{19}$ The 3' region, 5' region and intron of human VEGF gene are all highly polymorphic. VEGF gene has at least 30 single nucleotide polymorphism sites. Some single nucleotide polymorphisms have higher frequencies, with 10 polymorphisms just in its promoter region. 20 Where, $+936 \mathrm{C} / \mathrm{T}$ at the 3 '-end of the promoter is associated with RNA splicing. ${ }^{21}$ Studies have shown that distribution of VEGF $936 \mathrm{C} / \mathrm{T}$ polymorphism is significantly different in patients with endometriosis and normal controls. 22 There was statistically significant difference in VEGF $936 \mathrm{C} / \mathrm{T}$ allele frequency between III-IV grade endometriosis patients and normal controls. There has been no report on the relationship between VEGF $936 \mathrm{C} / \mathrm{T}$ gene polymorphism and IVF-ET/ICSI treatment outcome. Our results showed that frequencies of CC genotype and $\mathrm{C}$ allele in the non-pregnant group were higher than those in the pregnant group. The difference was statistically significant. This suggests that VEGF $936 \mathrm{C} / \mathrm{T}$ polymorphism may be associated with IVF-ET/ICSI treatment outcomes. Thus, using the combination of VEGF levels, embryo morphological parameters and VEGF $936 \mathrm{C} / \mathrm{T}$ gene polymorphisms to select embryos may obtain embryos with optimal growth potential for transplantation and improve the success rate of pregnancy.

There are some limitations and shortages in this research; for example, this study is limited to only Chinese women. No comparison between the Chinese women and the Western population in VEGF $936 \mathrm{C} / \mathrm{T}$ gene polymorphisms. So further relevant researches need to continue.

\section{CONCLUSION}

VEGF level in the embryo culture medium can be used as one reference indicator for predicting embryo quality and pregnancy outcome. The VEGF936C/T gene polymorphism may be related to IVF-ET/ICSI treatment outcome, and $C$ allele may be a susceptibility gene for IVF-ET/ICSI failure.
Acknowledgement: The study was funded by Yantai key R\&D project of Shandong Province (No.2016WS028).

\section{REFERENCES}

1. Roskoski R Jr. Vascular endothelial growth factor (VEGF) and VEGF receptor inhibitors in the treatment of renal cell carcinomas. Pharmacol Res 2017; 120:116-32.

2. Levitas E, Huleihal M, Lunenfeld E, Gakman R, Friger M, Potashnik G. Variations in vascular endothelial growth factor levels during ovarian superovulation and reduction of ovarian hyperstimulation incidence in young women: a prospective study. Open J Obstetr Gynecol 2013; 3:296-300.

3. Li W, Man XY, Li C M, Chen JQ, Zhou J, Cai SQ, et al. VEGF induces proliferation of human hair follicle dermal papilla cells through VEGF-2-mediated activation of ERK. Exp Cell Res 2012; 318:1633-40.

4. Bassino E, Zanardi A, Gasparri F, Munaron L. Effects of the biomimetic peptide sh-polipeptide 9 (CG-VEGF) on cocultures of human hair follicle dermal papilla cells and microvascular endothelial cells. Exper Dermatol 2016; 25:237-9.

5. Xu X, Du C, Li H, Du J, Yan X, Peng L, et al. Association of VEGF genetic polymorphisms with recurrent spontaneous abortion risk: a systematic review and meta-analysis. Plos One 2015; 10:e0123696.

6. Shim SH, Kim JO, Jeon YJ, An HJ, Lee HA, Kim JH, et al. Association between vascular endothelial growth factor promoter polymorphisms and the risk of recurrent implantation failure. Exper Therapeut Med 2018; 15:2109-19.

7. Jasensky J, Swain JE. Peering beneath the surface: novel imaging techniques to noninvasively select gametes and embryos for ART. Biol Reprod 2013; 89:105.

8. Sanchez T, Seidler EA, Gardner DK, Needleman D, Sakkas D. Will noninvasive methods surpass invasive for assessing gametes and embryos? Fertil Steril 2017; 108:730-7.

9. Fesahat F, Montazeri F, Sheikhha MH, Saeedi H, Dehghani FR, Kalantar SM. Frequency of chromosomal aneuploidy in high quality embryos from young couples using preimplantation genetic screening. Int J Reprod Biomed (Yazd) 2017; 15:297304.

10. Siristatidis C, Dafopoulos K, Salamalekis G, Galazios G, Christoforidis N, Moustakarias T, et al. Administration of lowmolecular-weight heparin in patients with two or more unsuccessful IVF/ICSI cycles: A multicenter cohort study. Gynecol Endocrinol 2018; 34:747-51.

11. Desai N, Ploskonka S, Goodman LR, Austin C, Goldberg J, Falcone T. Analysis of embryo morphokinetics, multinucleation and cleavage anomalies using continuous time-lapse monitoring in blastocyst transfer cycles. Reprod Biol Endocrinol 2014; $12: 54$.

12. El-Helaly M, Balkhy HH, Vallenius L. Carpal tunnel syndrome among laboratory technicians in relation to personal and ergonomic factors at work. J Occup Health 2017; 59:513-20.

13. Foresta C, Ubaldi FM, Rienzi L, Franchin C, Pivato M, Romano S, et al. Early protein profile of human embryonic secretome. Front Biosci 2016; 21:620-34.

14. Youm J, Kim SK, Jee BC, Kim SH. Embryonic survival, development and cryoinjury of repeatedly vitrified mouse preimplantation embryos. Eur J Obstet Gynecol Reprod Biol 2017; 217:66-70. 
15. Ren K, Li X, Yan J, Huang G, Zhou S, Yang B, et al. Knockdown of p66shc by sirna injection rescues arseniteinduced developmental retardation in mouse preimplantation embryos. Reprod Toxicol 2014; 43:8-18.

16. Karabulut AK, Pratten MK. The growth promoting effects of BFGF, PD-ECGF and, VEGF on cultured postimplantation rat embryos deprived of, serum fractions. J Anat 2000; 197:207-19.

17. Gao J, Gu F, Miao BY, Chen MH, Zhou CQ, Xu YW. Effect of the initiation of progesterone supplementation in in vitro fertilization-embryo transfer outcomes: A prospective randomized controlled trial. Fertil Steril 2017; 109:97-103.

18. Keshavarzi F, Salimi S, Mohammadpour-gharehbagh A, Teimoori B, Yazdi A, Farajianmashhadi $F$, et al. The -2549 insertion/deletion polymorphism of VEGF gene associated with uterine leiomyoma susceptibility in women from southeastern iran. Ginekol Pols 2017; 88:115-9.

19. Binder NK, Evans J, Gardner DK, Salamonsen LA, Hannan
NJ. Endometrial signals improve embryo outcome: functional role of vascular endothelial growth factor isoforms on embryo development and implantation in mice. Hum Reprod 2014; 29:2278-86.

20. Naikoo NA, Afroze D, Rasool R, Shah S, Ahangar AG, Bhat IA, et al. Snp and haplotype analysis of vascular endothelial growth factor (VEGF) gene in lung cancer patients of kashmir. Asian Pac J Cancer Prev 2017; 18:1799-804.

21. Kim OJ, Hong SH, Oh SH, Kim TG, Min KT, Oh D, et al. Association between VEGF polymorphisms and homocysteine levels in patients with ischemic stroke and silent brain infarction. Stroke 2011; 42:2393-402.

22. Cosín R, Gilabertestellés J, Ramón LA, España F, Gilabert J, Romeu A, et al. Vascular endothelial growth factor polymorphisms (-460C/T, $+405 \mathrm{G} / \mathrm{C}$, and $936 \mathrm{C} / \mathrm{T}$ ) and endometriosis: their influence on vascular endothelial growth factor expression. Fertil Steril 2009; 92:1214-20.

....占.... 